\title{
Intercarrier Compensation between Providers of Different Layers: Advantages of Transmission Initiator Determination
}

\author{
Ruzana Davoyan and Wolfgang Effelsberg \\ Department of Mathematics and Computer Science, \\ University of Mannheim, Germany \\ \{ruzana.davoyan, wolfgang.effelsberg\}@informatik.uni-mannheim.de
}

\begin{abstract}
This paper addresses the important issue of providing balanced allocation of the interconnection costs between networks. We analyze how beneficial is the determination of the original initiator of a transmission to the providers of different layers. The introduced model, where intercarrier compensation is based on the differentiated traffic flows, was compared with the existing solution, which performs cost compensation based on the traffic flows. For our analysis we considered both unilateral and bilateral settlement arrangements.
\end{abstract}

Keywords: Interconnection arrangement, intercarrier compensation, Internet economics.

\section{Introduction}

The Internet is a system of interconnected networks, which are connected either through a direct link or an intermediate point to exchange traffic. Currently, the Internet provides two basic types of interconnections such as peering and transit, and their variations. Peering is the arrangement of traffic exchange on the freesettlement basis, so that Internet service providers (ISPs) do not pay each other and derive revenues from their own customers. In transit, a customer ISP pays a transit ISP to deliver the traffic between the customers. Emergence of new types of ISPs (with large number of customers and great amount of content) led to appearance of new types of models, such as paid peering and partial transit.

Traditionally, before interconnecting, provider calculates whether the interconnection benefits would outweigh the costs [1]. Simple economic principle suggests sharing the costs between all parties. In the case of telephony, the study [2] argued that both calling and called parties benefit from the call, and consequently, should share the interconnection costs. In the Internet, under symmetry of traffic flows, the termination costs are set to zero, since it is assumed that the termination fees are roughly the same, and a peering arrangement is used. However, because no termination cost is charged, settlement-free model is considered inefficient in terms of cost compensation [3]. Generally, if providers are asymmetric in terms of size, peering is not appropriate, since providers incur different 
costs and benefit differently. In such a case, an interconnection arrangement is governed by the financial compensation in a bilaterally or unilaterally negotiated basis. In the bilateral settlement arrangements, the payments are based on the net traffic flow. In the unilateral settlement arrangements, a customer provider pays for sent and received traffic, even though traffic flows in both directions. This causes the existence of imbalance in allocation of the interconnection costs. In particular, smaller providers in high cost areas admit higher subscription fees. There exists a large body of literature that discusses interconnection challenges [1, 4-6]. Various pricing schemes have attempted to provide sustainable conditions for smaller ISPs [7-8]. These models make different trade-offs between the two objectives of interconnection pricing, viz., competition development and profitability. Hence, no single model has a clear advantage over the others. As cited in [9], it was recommended to establish bilateral settlement arrangements and to compensate each provider for the costs that it incurs in carrying traffic generated by the other network. However, it was argued that traffic flows are not a reasonable indicator to share the costs, since it is not clear who originally initiated a transmission and, therefore, who should pay for the costs. In other words, compensation between providers cannot be solely done based on the traffic flows, which provide a poor basis for cost sharing [9]. Recently provided analytical studies in [10-11], investigated the impact of the original initiator of a transmission at the wholesale and retail levels in the case of private peering arrangements. Further, we extended studies by examining the benefits of the customer providers only, which purchased transit services [12]. However, the remaining literature on the economics of interconnection considers the intercarrier compensation based only on the flows of traffic [2, 13-16].

The main objective of this paper is to explore the role of the determination of a transmission initiator on ISPs of different layers. The paper differs from the prior reported studies in that it considers how beneficial is the traffic differentiation to all providers, such as transit and customer, and it examines customer ISPs, which operate in different cost areas. Our studies involve the earlier introduced model, called Differentiated Traffic-based Interconnection Agreement (DTIA) that distinguishes traffic into two types to determine the transmission initiator in the IP networks and to compensate the costs. In contrast to the existing solutions [17], in which the payments are based on traffic flows (TF), we compensate differently for a particular traffic type. Unlike telephony, where the transmission initiator covers the entire costs, imposing uniform retail pricing, the proposed model distributes the joint costs between all parties and supports the diversity of existing retail pricing schemes in the Internet. Comparative studies were provided for the agreements based on the traffic flows and differentiated traffic flows compensations. We considered both unilateral and bilateral settlement models. The rest of the paper is organized as follows. Section 2 discusses existing financial settlements. Section 3 describes the motivation for traffic differentiation. Section 4 provides analytical studies. Section 5 concludes this paper. 


\section{Financial Settlements}

Generally, providers arrange financial settlements in order to determine the distribution of the interconnection costs [17]. Before examining financial settlements within the Internet, we consider the telephone system. As an example, assume a scenario, where Alice makes a call to Bob. Accepting the call, Bob incurs termination costs to its provider that should be covered either directly by billing Bob or indirectly by billing the calling party's carrier. As cited in [3], "existing access charge rules and the majority of existing reciprocal compensation agreements require the calling party's carrier, [...], to compensate the called party's carrier for terminating the call". Thus, the initiator of the call, i.e., Alice pays to a subscribed provider for the entire call since Alice asked to reserve the circuit. In contrast to the telephony example, establishing a connection in the Internet does not require any reservation of a circuit. Usually packets between Alice and Bob are routed independently, sometimes even via different paths. Therefore, as cited in [18], "it is very important to distinguish between the initiator and the sender, and likewise between the destination and the receiver". The initiator is the party that initiates a call or a session, and the destination is the party that receives a call. In contrast, the sender (the originator) is the party that sends traffic, and the receiver (the terminator) is the party that receives traffic. In telephony, the initiator is considered to be the originator and is charged based on the transaction unit, namely a "call minute" for using the terminating network. Even though it may be argued that a TCP session can be considered as a call, where the initiator of a session pays for the entire traffic flow, such a model deals with technical issues, considerable costs, and implies uniform retail pricing. Currently, the Internet uses the packet-based accounting model, under which the volume of the exchange traffic in both directions is measured, and adopts a small set of interconnection arrangements. Specifically, in the service-provider (unilateral) settlement, namely transit and paid peering business relationships, a customer ISP pays to a transit ISP for sent and received traffic. In the settlement-free agreement, namely peering relationships, providers do not pay each other. In some cases ISPs adopt the negotiated-financial (bilateral) settlement where the payments are based on the net flow of traffic. For detailed discussion see [17-19].

\section{Motivation for Traffic Differentiation}

The principle that we follow is that both parties derive benefits from the exchange of traffic and, therefore, should share the interconnection costs. Considering a system without externalities [20], the costs should be shared based on the benefits obtained by each party. However, in the real world, it is impossible to measure the benefits of parties and so to share the costs. If content is not equally distributed between providers, traffic imbalance occurs, and hence, costs and revenues are not shared evenly. As cited in [21], traffic flow is dominant towards a customer requested the content and generates $85 \%$ of the Internet traffic. This implies that inbound traffic is much more compared to outbound 
traffic of content request. In telephony for example, it is acceptable that more than $50 \%$ of rural network's revenue could come from the incoming calls. In contrast, in the Internet, customer networks pay for the entire traffic flows. It was recommended to compensate each provider for the costs that it incurs in carrying traffic based on the traffic flows. However, traffic flows are not a good measure for costs sharing, since "it is impossible to determine who originally initiated any given transmission on the Internet" and therefore, provide a poor basis for cost sharing [9]. On the other hand, providers are unwilling to inspect the IP header of a packet, since "the cost of carrying an individual packet is extremely small, and the cost of accounting for each packet may well be greater than the cost of carrying the packet across the providers" [19].

The DTIA model presented in [22] manages inter-provider cost compensation considering the original initiator of a transmission. In order to determine a party that originally initiated a transmission, we differentiated traffic into two types, referred to as native, which is originally initiated by the provider's own customers, and stranger that is originally initiated by the customers of any other network. Indeed, outgoing traffic of $\mathrm{ISP}_{i}$ that is the same as adjacent provider's incoming traffic may be i) either a part of a transmission initiated by a customer of $\mathrm{ISP}_{i}$, ii) or a part of a transmission initiated by a customer of any other network. Furthermore, we suggest that providers compensate differently for a particular type of traffic, where stranger traffic is charged at a lower rate than native traffic. More specifically, each provider settled DTIA compensates the cost of carrying traffic according to the differentiated traffic flows. For detailed description of the DTIA model and its traffic management mechanism see [22].

\section{The Model of Interconnection}

In our analysis we follow an assumption done in [13] to capture traffic imbalance and therefore, consider two types of the customers, namely websites (who host information and content) and consumers (who use information and content provided on websites). Actually, traffic is exchanged between consumers, between websites, from websites to consumers, and from consumers to websites. According to the proposed approach, a node (customer) in a P2P network is considered as a consumer as well as a website simultaneously, since it can act as a client and as a server. Thus, traffic generated from websites to consumers and vice versa along with Web, FTP and streaming media traffic captures P2P traffic. Traffic between consumers captures VoIP traffic that tends to be symmetric, and email exchange that is much smaller than traffic generated from websites to consumers. To focus on explicit monetary transfers between providers and traffic asymmetry in its simplest way, we consider traffic exchange i) from consumers to websites, and ii) from websites to consumers. To simplify analytical studies the following assumptions were made throughout the paper:

Assumption 1. Let $\alpha_{i} \in(0,1)$ network $i$ 's market share for consumers and $\beta_{i} \in(0,1)$ its market share for websites. The market consists of only one transit and two customer ISPs, $i$ and $j$, where $i \neq j=1,2$ and $\alpha_{i}+\alpha_{j}=\alpha, \beta_{i}+\beta_{j}=\beta$. 
Assumption 2. Balanced calling pattern, where each consumer requests any website in any network with the same probability is considered1. Each consumer originates one unit of traffic per each request of website. The number of consumers and websites in the market is given by $N$ and $M$ respectively.

We examine a scenario, in which $\mathrm{ISP}_{i}$ and $\mathrm{ISP}_{j}$ exchange traffic through the transit $\mathrm{ISP}_{k}$. The amount of the differentiated traffic originated from $\operatorname{ISP}_{i}$ with destination to $\operatorname{ISP}_{j}$ and vice versa is given by

$$
\begin{array}{ll}
t_{i k}^{n a t}=\alpha_{i} \beta_{j} N M & t_{j k}^{n a t}=\alpha_{j} \beta_{i} N M \\
t_{i k}^{s t r}=\alpha_{j} \beta_{i} N M x & t_{j k}^{s t r}=\alpha_{i} \beta_{j} N M x
\end{array}
$$

where $t_{i k}^{n a t}\left(t_{j k}^{n a t}\right)$ denotes the amount of outgoing native traffic (exchanged from consumers to websites) and $t_{i k}^{s t r}\left(t_{j k}^{s t r}\right)$ is the amount of stranger traffic (exchanged from websites to consumers) with respect to $\operatorname{ISP}_{i}\left(\operatorname{ISP}_{j}\right)$. The variable $x$ denotes the average amount of traffic caused by requesting a website. It is known that $\mathrm{P} 2 \mathrm{P}$ traffic asymmetry is typically caused by less capacity provisioned in the upstream direction. Thus, upstream/downstream P2P traffic flows can be asymmetric, which implies that $x$ is different for the customers subscribed to different ISPs. However, this does not affect the results of our studies. The total amount of traffic originated by $\mathrm{ISP}_{i}$ and $\mathrm{ISP}_{j}$ are $t_{i k}=t_{i k}^{n a t}+t_{i k}^{s t r}$ and $t_{j k}=t_{j k}^{n a t}+t_{j k}^{s t r}$.

\subsection{Unilateral Settlement Arrangements}

We start by considering a unilateral settlement arrangement, where transit ISP charges the customer providers for every unit of traffic sent and received. Let $c_{i}^{k}$ and $c_{j}^{k}$ are the marginal costs of the connectivity of $\operatorname{ISP}_{i}$ and $\operatorname{ISP}_{j}$ correspondingly. These providers operate in different $\operatorname{cost}$ areas so that $c_{i}^{k}<c_{j}^{k}$, where marginal costs exhibit increasing returns to scale (i.e., $\mathrm{ISP}_{i}>\mathrm{ISP}_{j}$ ). $\mathrm{ISP}_{k}$ charges the customer ISPs $a_{k}$ and $b_{k}$ for every unit of native and stranger traffic respectively, where $a_{k}>b_{k}$ (ISPs pay less for stranger traffic). In particular, in DTIA we consider that a customer ISP i) compensates fully the imbalance in the connectivity costs between endpoints, if the exchanged traffic is native, and ii) does not compensate this difference, if the originated traffic is stranger. The difference in the costs of the exchanged traffic between the points is defined by

$$
\Delta=c_{j}^{k}-c_{i}^{k}
$$

Proposition 1. The access charge for stranger traffic is set to the lowest cost of the connectivity, i.e., $b_{k}=c_{i}^{k}$.

Proof. Interconnection costs between the customer providers are covered by the access charges. Since native traffic for $\mathrm{ISP}_{i}$ is stranger for $\mathrm{ISP}_{j}$, the sum of fees for native and stranger traffic are equal to the whole costs, that is

$$
c_{i}^{k}+c_{j}^{k}=a_{k}+b_{k}
$$

${ }^{1}$ Due to the lack of mathematical models on how traffic between ISPs is distributed, many works make a statistical assumption, such as balanced calling pattern. 
In the DTIA model, a provider compensates the imbalance in the costs expressed by (3) fully only for native traffic. This cost difference is not compensated for the stranger traffic. Consequently, it can be written that

$$
a_{k}=b_{k}+\Delta
$$

By substituting (3) and (5) in (4), it can be obtained that access rate for stranger traffic is set to the lowest cost of the connectivity, that is $b_{k}=c_{i}^{k}$. Obviously, that the access charge for native traffic is defined by $a_{k}=c_{i}^{k}+\Delta$.

We investigate the payments of the customer providers in the classical and DTIA models. The payments of $\mathrm{ISP}_{i}$ and $\mathrm{ISP}_{j}$ to transit ISP in DTIA are given by

$$
\begin{aligned}
& f_{i k}=a_{k}\left(t_{i k}^{n a t}+t_{j k}^{s t r}\right)+b_{k}\left(t_{i k}^{s t r}+t_{j k}^{n a t}\right) \\
& f_{j k}=a_{k}\left(t_{j k}^{n a t}+t_{i k}^{s t r}\right)+b_{k}\left(t_{j k}^{s t r}+t_{i k}^{n a t}\right)
\end{aligned}
$$

The sum of these payments presents the incremental revenue of the transit ISP $\pi_{k}=f_{i k}+f_{j k}$. The net payments of the customer ISPs according to the traffic flow based compensation are denoted by $\check{f}_{i k}$ and $\check{f}_{j k}$ and calculated as follows

$$
\begin{aligned}
& \check{f}_{i k}=c_{i}^{k}\left(t_{i k}+t_{j k}\right) \\
& \check{f}_{j k}=c_{j}^{k}\left(t_{i k}+t_{j k}\right)
\end{aligned}
$$

Proposition 2. The payments of larger (smaller) providers are higher (less) in DTIA than these in the classical model.

Proof. Considering the net payments of the larger $\mathrm{ISP}_{i}$, from (6) and (8) follows that $\check{f}_{i k}-f_{i k}=\left(b_{k}-a_{k}\right)\left(t_{i k}^{n a t}+t_{j k}^{s t r}\right)<0$, i.e., $f_{i k}>\check{f}_{i k}$. Similarly, comparing the payments of ISP $_{j}$ in the DTIA and classical models given by (7) and (9) we obtain that $\check{f}_{j k}-f_{j k}=\left(a_{k}-b_{k}\right)\left(t_{i k}^{n a t}+t_{j k}^{s t r}\right)>0$. This gives that $f_{j k}<\check{f}_{j k}$.

\subsection{Bilateral Settlement Arrangements}

This subsection examines bilateral settlement models, where each provider (including customer ISP) is compensated for the costs of carrying traffic.

Reciprocal Access Charges. In the following lines we explore the case when the customer providers charge the transit provider reciprocal access charges and vice versa. Let $b$ be the access payment that $\operatorname{ISP}_{k}$ subsidizes $\mathrm{ISP}_{i}$ and $\mathrm{ISP}_{j}$ for every unit of sent traffic, where $b<c_{j}^{k}$. The marginal connectivity costs of the customer providers charged by $\mathrm{ISP}_{k}$ can be written as follows

$$
c_{i}^{k}+c_{j}^{k}=c_{k}+\sigma
$$

where $c_{k}$ is the marginal transportation cost of $\mathrm{ISP}_{k} ; \sigma$ is an arbitrary constant.

Proposition 3. The access charge for stranger traffic set by $I S P_{k}$ is equal to $b_{k}=c_{k}+b$ (i.e., the total costs of $I S P_{k}$ ). 
Proof. The costs of $\mathrm{ISP}_{k}$ are comprised of the marginal transmission cost and the payment to access customer provider's infrastructure, i.e., $c_{k}+b$. The bilateral settlement model is attractive to $\mathrm{ISP}_{k}$ only if its own costs are covered. These costs correspond to the minimum level of access charge set by $\mathrm{ISP}_{k}$, that is

$$
c_{k}+b=\min \left\{a_{k}, b_{k}\right\}
$$

In DTIA provider compensates less the costs of carrying stranger traffic, thus

$$
b_{k}=c_{k}+b
$$

Obviously, that the access charge for native traffic set by the transit provider is increased by the arbitrary constant and calculated as follows $a_{k}=b_{k}+\sigma$.

The net interconnection payments from $\operatorname{ISP}_{i}$ and $\operatorname{ISP}_{j}$ to $\mathrm{ISP}_{k}$ are defined by

$$
f_{i k}=a_{k} t_{i k}^{n a t}+b_{k} t_{i k}^{s t r} \quad f_{j k}=a_{k} t_{j k}^{n a t}+b_{k} t_{j k}^{s t r}
$$

Analogously, the net transfers of $\mathrm{ISP}_{k}$ to the customer providers are given by

$$
f_{k i}=b\left(t_{j k}^{n a t}+t_{j k}^{s t r}\right) \quad f_{k j}=b\left(t_{i k}^{n a t}+t_{i k}^{s t r}\right)
$$

It can be noticed that the transit ISP is charged based on the rate for stranger traffic, because we consider that it does not have any customers of its own.

Before examining the payments of the customer ISPs in the DTIA and classical models with bilateral settlements, we consider access charges and net payments in the classical solution. Let $\check{b}$ be the payment paid by $\operatorname{ISP}_{k}$ to the customer providers for sending traffic. In the model with bilateral settlements, the access charge set by the transit provider is defined by $\check{a}_{k}=c_{i}^{k}+c_{j}^{k}+\check{b}$. Assume that $\mathrm{ISP}_{k}$ has users, thus $b$ (in DTIA) is the rate charged by the customer ISPs for unit of stranger traffic only, while $\breve{b}$ (in the classical model) is payment for unit of traffic. As a result, we obtain that $\check{b} \geq b$. The payments of $\operatorname{ISP}_{i}$ and $\operatorname{ISP}_{j}$ are

$$
\check{f}_{i k}=\check{a}_{k} t_{i k} \quad \check{f}_{j k}=\check{a}_{k} t_{j k}
$$

Proposition 4. The net payments of the customer providers in the DTIA model are less than these in the classical model.

Proof. Considering the payments of $\mathrm{ISP}_{i}$ and $\mathrm{ISP}_{j}$ given by (12) and (14) follows

$$
\begin{aligned}
& \check{f}_{i k}-f_{i k}=t_{i k}^{n a t}\left(\check{a}_{k}-a_{k}\right)+t_{i k}^{s t r}\left(\check{a}_{k}-b_{k}\right)>0 \\
& \check{f}_{j k}-f_{j k}=t_{j k}^{n a t}\left(\check{a}_{k}-a_{k}\right)+t_{j k}^{s t r}\left(\check{a}_{k}-b_{k}\right)>0
\end{aligned}
$$

Non-reciprocal Access Charges. We continue with examination of the bilateral settlement model with asymmetric access fees. Let $b_{i}$ and $b_{j}\left(b_{i}<b_{j}\right)$ are the access rates for every unit of traffic received by $\operatorname{ISP}_{i}$ and $\operatorname{ISP}_{j}$ correspondingly. 
Following the results of Proposition 3, fees that the transit provider charges the customer ISPs for native and stranger traffic can be rewritten as

$$
\begin{aligned}
a_{i k} & =c_{i}^{k}+c_{j}^{k}+b_{j} & a_{j k} & =c_{i}^{k}+c_{j}^{k}+b_{i} \\
b_{i k} & =c_{k}+b_{j} & b_{j k} & =c_{k}+b_{i}
\end{aligned}
$$

The net payments from $\operatorname{ISP}_{i}$ and $\mathrm{ISP}_{j}$ to the transit $\mathrm{ISP}_{k}$ and vice versa are

$$
\begin{array}{ll}
f_{i k}=a_{i k} t_{i k}^{n a t}+b_{i k} t_{i k}^{s t r} & f_{j k}=a_{j k} t_{j k}^{n a t}+b_{j k} t_{j k}^{s t r} \\
f_{k i}=b_{i}\left(t_{j k}^{n a t}+t_{j k}^{s t r}\right) & f_{k j}=b_{j}\left(t_{i k}^{n a t}+t_{i k}^{s t r}\right)
\end{array}
$$

The following lines explore the payments of customer ISPs in the classical and DTIA models. For this purpose, we consider access charges and payments in the classical solution. The access rates that $\mathrm{ISP}_{k}$ charges $\mathrm{ISP}_{i}$ and $\mathrm{ISP}_{j}$ are

$$
\check{a}_{i k}=c_{i}^{k}+c_{j}^{k}+\check{b}_{j} \quad \check{a}_{j k}=c_{i}^{k}+c_{j}^{k}+\check{b}_{i}
$$

where $\check{b}_{i}$ and $\check{b}_{j}\left(\check{b}_{i} \geq b_{i}, \check{b}_{j} \geq b_{j}\right)$ are access fees set by the customer providers correspondingly. The net payments of the customer providers are given by

$$
\check{f}_{i k}=\check{a}_{i k} t_{i k} \quad \check{f}_{j k}=\check{a}_{j k} t_{j k}
$$

Proposition 5. The interconnection payments of the customer providers are less in DTIA than these in the classical model.

Proof. Examining the payments of $\mathrm{ISP}_{i}$ and $\mathrm{ISP}_{j}$ given by (17) and (19) follows

$$
\begin{gathered}
\check{f}_{i k}-f_{i k}=t_{i k}^{n a t}\left(\check{a}_{i k}-a_{i k}\right)+t_{i k}^{s t r}\left(\check{a}_{i k}-b_{i k}\right)>0 \\
\check{f}_{j k}-f_{j k}=t_{j k}^{n a t}\left(\check{a}_{j k}-a_{j k}\right)+t_{j k}^{s t r}\left(\check{a}_{j k}-b_{j k}\right)>0
\end{gathered}
$$

\subsection{Discussions}

Tables 1,3 report the results of analytical studies, which examined how beneficial is the determination of a transmission initiator to the providers of different layers. The comparison results between unilateral settlement models are presented in Table 1. Tables 2 and 3 demonstrate the comparison of bilateral settlement arrangements with symmetric and asymmetric access charges. The analyses considered all available market states in terms of providers' market shares, where $\mathrm{ISP}_{i}>\mathrm{ISP}_{j}$. The following parameter values were imposed to calculated the specific outcomes: $c_{i}^{k}=0.4, c_{j}^{k}=1.5, c_{k}=0.9, b=0.5, b_{i}=0.3, b_{j}=0.5$, $x=35, N=100$, and $M=60$. In order to simplify analyses we assume that $\check{b}=b, \breve{b}_{i}=b_{i}$, and $\check{b}_{j}=b_{j}$. The parameters are chosen to satisfy a condition that providers operate in different cost areas. However, the specification is clearly arbitrary. It is important to note, that our conclusions do not heavily depend on the chosen parameter values. The results obtained for a number of other parameter sets have not produced significant changes. Network $i$ 's total incremental 
Table 1. Comparative Results of DTIA with Unilateral Settlements

\begin{tabular}{|c|c|c|c|c|c|c|c|c|c|c|c|c|}
\hline \multirow[t]{2}{*}{$\overline{\overline{\text { Case }}}$} & \multirow[t]{2}{*}{$\overline{\alpha_{i}}$} & \multirow[t]{2}{*}{$\overline{\bar{\beta} \beta_{i}}$} & \multirow[t]{2}{*}{$\overline{\overline{t_{i k}^{n a t}}}$} & \multirow[t]{2}{*}{$\overline{\overline{t_{j k}^{n a t}}}$} & \multirow[t]{2}{*}{$\overline{t_{i k}}$} & \multirow[t]{2}{*}{$\overline{\overline{t_{j k}}}$} & \multicolumn{2}{|c|}{$\overline{\overline{f_{i k}}}$} & \multicolumn{2}{|c|}{$\overline{\overline{f_{j k}}}$} & \multicolumn{2}{|c|}{$\overline{\pi_{k}}$} \\
\hline & & & & & & & TIA & TF & DTIA & TF & DTIA & TF \\
\hline I & 0.5 & 0.9 & 300 & 2700 & 800 & 13200 & 55080 & 43200 & 150120 & $\overline{62000}$ & 05 & $\overline{05200}$ \\
\hline$\alpha_{i}=\alpha_{j}$ & 0.5 & 0.8 & 600 & 2400 & 84600 & 23400 & 66960 & 43200 & 138240 & 162000 & 20520 & 205200 \\
\hline$\beta_{i}>\beta_{j}$ & 0.5 & 0.7 & 900 & 2100 & 74400 & 33600 & 78840 & 43200 & 126360 & 1620 & 2052 & 205200 \\
\hline$\overline{\text { II }}$ & 0.9 & 0.5 & 2700 & 300 & 13200 & 94800 & 150120 & 43200 & 55080 & 162000 & 205200 & 205200 \\
\hline$\alpha_{i}>\alpha_{j}$ & 0.8 & 0.5 & 2400 & 600 & 23400 & 84600 & 138240 & 43200 & 66960 & 1620 & & 5200 \\
\hline$\beta_{i}=\beta_{j}$ & 0.7 & 0.5 & 2100 & 900 & 33600 & 74400 & 126360 & 43200 & 78840 & 16200 & 205 & 205200 \\
\hline$\overline{\text { III }}$ & 0.9 & 0.8 & 1080 & 480 & 17880 & 38280 & 65232 & 22464 & 41472 & 84240 & 106704 & 106704 \\
\hline$\alpha_{i}>\alpha_{j}$ & 0.8 & 0.7 & 1440 & 840 & 30840 & 51240 & 89856 & 32832 & 66096 & 123120 & 155952 & 155952 \\
\hline$\beta_{i}>\beta_{j}$ & 0.7 & 0.6 & 1680 & 1080 & 39480 & 59880 & $10627^{\circ}$ & 39744 & 82512 & 149040 & 887 & 188784 \\
\hline$\alpha_{i}>\beta_{i}$ & 0.6 & 0.55 & 1620 & 1320 & 47820 & 58020 & 106488 & 42336 & 94608 & 158760 & 201096 & 201096 \\
\hline$\overline{\mathrm{IV}}$ & 0.9 & 0.9 & 540 & 540 & 19440 & 19440 & 36936 & 15552 & 36936 & 58320 & 73872 & 73872 \\
\hline$\alpha_{i}>\alpha_{j}$ & 0.8 & 0.8 & 960 & 960 & 34560 & 34560 & 65664 & 27648 & 65664 & 103680 & 131328 & 131328 \\
\hline$\beta_{i}>\beta_{j}$ & 0.7 & 0.7 & 1260 & 1260 & 45360 & 45360 & 86184 & 36288 & 86184 & 136080 & 172368 & 172368 \\
\hline$\alpha_{i}=\beta_{i}$ & 0.6 & 0.6 & 1440 & 1440 & 51840 & 51840 & 98496 & 41472 & 98496 & 155520 & 196992 & 196992 \\
\hline$\overline{\mathbf{V}}$ & 0.9 & 0.2 & 4320 & 120 & 8520 & 15132 & 23500 & 63936 & 68688 & 2397 & 30369 & 303696 \\
\hline & 0.8 & 0.25 & 3600 & 300 & 14100 & 126300 & 19872 & 56160 & 68040 & 210600 & 26676 & 266760 \\
\hline$\beta_{i}<\beta_{j}$ & 0.7 & .35 & 2730 & 630 & 24780 & 96180 & 156492 & 48384 & 73332 & 181440 & 229824 & 229824 \\
\hline
\end{tabular}

Table 2. Comparative Results of Bilateral Settlement Arrangements (Reciprocal Access Charges)

\begin{tabular}{lcccccccccccc}
\hline Case & \multicolumn{2}{c}{$f_{i k}$} & \multicolumn{2}{c}{$f_{j k}$} & $f_{k i}$ & $f_{k j}$ & \multicolumn{2}{c}{$r_{k}$} & \multicolumn{3}{c}{$r_{i}$} & \multicolumn{3}{c}{$r_{j}$} \\
& DTIA & TF & DTIA & TF & & & DTIA & TF & DTIA & TF & DTIA & TF \\
\hline I & 133020 & 227520 & 21180 & 31680 & 6600 & 47400 & 100200 & 205200 & 126420 & 220920 & -26220 & -15720 \\
& 119040 & 203040 & 35160 & 56160 & 11700 & 42300 & 100200 & 205200 & 107340 & 191340 & -7140 & 13860 \\
& 105060 & 178560 & 49140 & 80640 & 16800 & 37200 & 100200 & 205200 & 88260 & 161760 & 11940 & 43440 \\
\hline $\mathbf{I I}$ & 21180 & 31680 & 133020 & 227520 & 47400 & 6600 & 100200 & 205200 & -26220 & -15720 & 126420 & 220920 \\
& 35160 & 56160 & 119040 & 203040 & 42300 & 11700 & 100200 & 205200 & -7140 & 13860 & 107340 & 191340 \\
& 49140 & 80640 & 105060 & 178560 & 37200 & 16800 & 100200 & 205200 & 11940 & 43440 & 88260 & 161760 \\
\hline $\mathbf{I I I}$ & 26112 & 42912 & 54072 & 91872 & 19140 & 8940 & 52104 & 106704 & 6972 & 23772 & 45132 & 82932 \\
& 44616 & 74016 & 72576 & 122976 & 25620 & 15420 & 76152 & 155952 & 18996 & 48396 & 57156 & 107556 \\
& 56952 & 94752 & 84912 & 143712 & 29940 & 19740 & 92184 & 188784 & 27012 & 64812 & 65172 & 123972 \\
& 68568 & 114768 & 82548 & 139248 & 29010 & 23910 & 98196 & 201096 & 39558 & 85758 & 58638 & 115338 \\
\hline $\mathbf{I V}$ & 27756 & 46656 & 27756 & 46656 & 9720 & 9720 & 36072 & 73872 & 18036 & 36936 & 18036 & 36936 \\
& 49344 & 82944 & 49344 & 82944 & 17280 & 17280 & 64128 & 131328 & 32064 & 65664 & 32064 & 65664 \\
& 64764 & 108864 & 64764 & 108864 & 22680 & 22680 & 84168 & 172368 & 42084 & 86184 & 42084 & 86184 \\
& 74016 & 124416 & 74016 & 124416 & 25920 & 25920 & 96192 & 196992 & 48096 & 98496 & 48096 & 98496 \\
\hline $\mathbf{V}$ & 16248 & 20448 & 211968 & 363168 & 75660 & 4260 & 148296 & 303696 & -59412 & -55212 & 207708 & 358908 \\
& 23340 & 33840 & 177120 & 303120 & 63150 & 7050 & 130260 & 266760 & -39810 & -29310 & 170070 & 296070 \\
& 37422 & 59472 & 135282 & 230832 & 48090 & 12390 & 112224 & 229824 & -10668 & 11382 & 122892 & 218442 \\
\hline \hline
\end{tabular}


Table 3. Comparative Results of Bilateral Settlement Models (Non-reciprocal Access Charges)

\begin{tabular}{|c|c|c|c|c|c|c|c|c|c|c|c|c|}
\hline \multirow[t]{2}{*}{$\overline{\text { Case }}$} & \multicolumn{2}{|c|}{$f_{i k}$} & \multicolumn{2}{|c|}{$f_{j k}$} & \multirow[t]{2}{*}{$f_{k i}$} & \multirow[t]{2}{*}{$f_{k j}$} & \multicolumn{2}{|c|}{$r_{k}$} & \multicolumn{2}{|c|}{$r_{i}$} & \multicolumn{2}{|c|}{$r_{j}$} \\
\hline & DTIA & TF & TIA & TF & & & DTIA & TF & DTIA & TF & DTIA & TF \\
\hline & 133020 & 227520 & 18540 & 29040 & 3960 & 47400 & 100200 & 205200 & 129060 & 223560 & -28860 & -18360 \\
\hline & 11 & 10 & 30480 & 51480 & 7020 & 42300 & & 05200 & 11 & 1 & -11820 & 9180 \\
\hline & 105060 & 178560 & 42420 & 73920 & 10080 & 37200 & 100 & 205200 & 94980 & 168480 & 5220 & 36720 \\
\hline \multirow[t]{3}{*}{$\overline{\text { II }}$} & 21180 & 31680 & 114060 & 208560 & 28440 & 6600 & 100200 & 205200 & -7260 & 3240 & 107460 & 201960 \\
\hline & 60 & 6160 & 02120 & 186120 & 25380 & 00 & & 0 & 9780 & 0780 & 20 & 174 \\
\hline & 49140 & 80640 & 90180 & 163680 & 22320 & 16800 & 100200 & 205200 & 26820 & 58320 & 73380 & 146880 \\
\hline \multirow[t]{4}{*}{ IIII } & 26112 & 42912 & 46416 & 84216 & 11484 & 8940 & 52104 & 106704 & 14628 & 31428 & 37476 & 75276 \\
\hline & 44616 & 74016 & 62328 & 112728 & 15372 & 15420 & 76152 & 155952 & 29244 & 58644 & 46908 & 97308 \\
\hline & 56952 & 94752 & 72936 & 131736 & 17964 & 19740 & 92184 & 188784 & 38988 & 76788 & 53196 & 111996 \\
\hline & 68568 & 114768 & 70944 & 127644 & 17406 & 23910 & 98196 & 201096 & 51162 & 97362 & 47034 & 103734 \\
\hline \multirow[t]{4}{*}{$\overline{\mathbf{I V}}$} & 27756 & 46656 & 23868 & 42768 & 5832 & 9720 & 36072 & $\begin{array}{l}73872 \\
\end{array}$ & 21924 & 40824 & 14148 & 33048 \\
\hline & 49344 & 82944 & 42432 & 76032 & 10368 & 17280 & 64128 & 131328 & 38976 & 72576 & 25152 & 58752 \\
\hline & 64764 & 108864 & 55692 & 99792 & 13608 & 22680 & 84168 & 172368 & 51156 & 95256 & 33012 & 77112 \\
\hline & 74016 & 124416 & 63648 & 114048 & 15552 & 25920 & 96192 & 196992 & 58464 & 108864 & 37728 & 88128 \\
\hline \multirow[t]{3}{*}{$\mathbf{V}$} & 16248 & 20448 & 181704 & 332904 & 45396 & 4260 & 148296 & 303696 & -29148 & -24948 & 177444 & 328644 \\
\hline & 340 & 33840 & 15186 & 277 & 37890 & 7050 & 130260 & 266760 & -14550 & -4050 & 144 & 270810 \\
\hline & 37422 & 59472 & 116046 & 211596 & 28854 & 12390 & 112224 & 229824 & 8568 & 30618 & 103656 & 199206 \\
\hline
\end{tabular}

cost of connectivity is defined by $r_{i}=f_{i k}-f_{k i}$. Network $k$ 's profit obtained from interconnection is $r_{k}=\left(f_{i k}+f_{j k}\right)-\left(f_{k i}+f_{k j}\right)$.

Comparative results obtained for arrangements with unilateral settlements demonstrated that in the presented model the payments are decreased for the smaller ISP and are increased for the larger ISP. This is achieved by different access rates charged for the distinguished traffic flows. Specifically, the payments of $\mathrm{ISP}_{i}$ are increased due to native traffic compensation, while the payments of $\mathrm{ISP}_{j}$ are decreased due to stranger traffic compensation. Further, the results showed that in DTIA the more outgoing traffic the lower costs of the provider. In particular, incoming and outgoing native traffic are directly proportional. Hence, the network that sends more native traffic incurs higher costs than the network that receives this traffic. This is explained by the higher access charges for native than stranger traffic. The costs of both customer ISPs are equal only in the case when their native and stranger traffic volumes are symmetric correspondingly. Finally, the results indicated that the revenues of the transit provider in the classical model based on the traffic flows compensation and DTIA are equal.

The key consequences provided below are based on the analytical studies, which explored bilateral settlement arrangements. In DTIA, the payments paid by the customer providers are decreased and these of transit provider remain the same. Specifically, providers $\mathrm{ISP}_{i}$ and $\mathrm{ISP}_{j}$ compensate based on the differentiated traffic flows, where the access charge for stranger traffic flow is lower than the access charge set in the classical model. As a consequence, the total incremental costs of the customer providers $\left(r_{i}\right.$ and $\left.r_{j}\right)$ are also decreased. On the 
other side, profits of $\mathrm{ISP}_{k}$ obtained from the interconnection (i.e., the differences between received and paid payments, $r_{k}$ ) are lower than these in the traffic flow based compensation model. However, as mentioned earlier, it was argued that traffic flows provide a poor basis for Internet interconnection cost sharing.

The provided studies examined a model consisting of one transit and two customer ISPs. One question that arises here is on the robustness of the obtained results for more realistic scenarios, which consider more transit and customer ISPs. From Propositions 2, 4 and 5 it can be noticed that the results depend only on the access charges of both DTIA and classical models. More specifically, in the unilateral settlement arrangements, the results rely on the inequality $\left(a_{k}-b_{k}\right)>$ 0 . Analogously, the results given by (15) and (16) depend on the inequalities $\left(\check{a}_{k}-a_{k}\right)>0$ and $\left(\check{a}_{k}-b_{k}\right)>0$, while results expressed by (20) and (21) are based on $\left(\check{a}_{i k}-a_{i k}\right)>0$ and $\left(\check{a}_{i k}-b_{i k}\right)>0$. Hence, the provided conclusions remain the same. Obviously, in the extended scenarios, access charges are obtained by solving a system of linear equations.

\section{$5 \quad$ Summary and Conclusions}

In this paper we proposed models and their analysis, which are based on the DTIA strategy for inter-provider cost compensation. The goal for this was to explore how the determination of a transmission initiator affects different providers, operated in different cost areas and arranged interconnection with unilateral and bilateral settlements (Tables 1,3). The results obtained from the analytical studies showed that DTIA was able to find better results (in terms of interconnection payments) than the classical solution for the both models. More specifically, the proposed scheme diminishes the existing inequity in allocation of the interconnection costs. From the comparison between unilateral settlement models follows that the costs of the smaller provider are decreased. This stimulates the retail prices fall in the market, where the provider operates and consequently, the development of the infrastructure in terms of subscribed customers. The growth of the smaller ISP leads to balance the volumes of a particular traffic type, and as a result, reduces the imbalance in cost allocation. Obviously, that revenue of the larger ISP obtained from retail market will be increased. From the perspective of a transit provider, its revenues obtained from the customer providers remain the same in the DTIA and classical models.

In the bilateral settlement arrangements, the net payments of both customer ISPs in the DTIA model are decreased. This leads to the decrease in the incremental revenue obtained by the transit provider. Finally, the comparison between the existing model with unilateral settlement and DTIA with bilateral settlement showed that our approach generally performed better for both smaller and larger ISPs in terms of reduced net payments. For the smaller provider, DTIA is dominated in all cases over the classical model, and for the larger provider only in Cases II and V. Obviously, that the profits of the transit provider in bilateral settlement model are decreased, since it along with other ISPs shares the interconnection costs. Resuming, the provision of a model, which compensates 
providers while exploiting their infrastructures, is advantageous for sustainable environment. From this point of view the proposed DTIA model is beneficial.

\section{References}

1. Laffont, J.J., Tirole, J.: Competition in telecommunications. MIT Press, Cambridge (2000)

2. DeGraba, P.: Bill and Keep at the central office as the efficient interconnection regime, FCC, OPP, Working Paper 33 (December 2000)

3. Federal Communications Commission: In the matter of developing a unified intercarrier compensation regime, Notice of Proposed Rulemaking (April 27, 2001)

4. Bezzina, J.: Interconnection challenges in a converging environment, the World Bank, Global Information and Communication Technologies Department (2005)

5. Dymond, A.: Telecommunications challenges in developing countries - asymmetric interconnection charges for rural areas, World Bank working paper (December 2004)

6. Lie, E.: International Internet interconnection next generation networks and development, GSR (2007)

7. Armstrong, M.: The theory of access pricing and interconnection. In: Handbook of Telecommunications Economics, vol. 1. North-Holland, Amsterdam (2002)

8. Noam, E.: Interconnection practices. In: Handbook of Telecommunications Economics, vol. 1. North-Holland, Amsterdam (2002)

9. Kende, M.: The digital handshake: connecting Internet backbones, FCC, OPP Working Paper No. 32 (September 2000)

10. Davoyan, R., Altmann, J.: Investigating the influence of market shares on interconnection settlements. In: Proceedings of IEEE Globecom (November 2008)

11. Davoyan, R., Altmann, J.: Investigating the role of a transmission initiator in private peering arrangements. In: Proceedings of IFIP/IEEE IM (June 2009)

12. Davoyan, R., Altmann, J., Effelsberg, W.: Intercarrier Compensation in Unilateral and Bilateral Arrangements. In: Proceedings of IEEE ICCCN (August 2009)

13. Laffont, J.J., Marcus, S., Rey, P., Tirole, J.: Internet interconnection and the offnet-cost pricing principle. RAND Journal of Economics (2003)

14. Weiss, M.B., Shin, S.: Internet Interconnection Economic Model and its Analysis: Peering and Settlement. Netnomics 6(1) (2004)

15. Besen, S., Milgrom, P., Mitchell, B., Srinagesh, P.: Advancing in routing technologies and Internet peering agreements. American Economic Review (2001)

16. Shrimali, G., Kumar, S.: Bill-and-Keep peering, in Telecom. Policy (2006)

17. Huston, G.: Interconnection, peering, and settlements, Part II. Internet Protocol Journal, Cisco Publications 2(2), 2-23 (1999)

18. Yoon, K.: Interconnection economics of all-IP networks. Review of Network Economics 5 (2006)

19. Huston, G.: Interconnection and peering (November 2000)

20. Economides, N.: The economics of networks. International Journal of Industrial Organization 14(6), 673-699 (1996)

21. Huston, G.: ISP survival guide: strategies for running a competitive ISP. Wiley, Chichester (1998)

22. Davoyan, R., Altmann, J., Effelsberg, W.: A new bilateral arrangement between interconnected providers. In: Reichl, P., Stiller, B., Tuffin, B. (eds.) ICQT 2009. LNCS, vol. 5539, pp. 85-96. Springer, Heidelberg (2009) 